\title{
Alterations in serum amino acid profiles in children with attention deficit/hyperactivity disorder
}

\author{
ANATOLY V. SKALNY ${ }^{1,2}$, ANNA L. MAZALETSKAYA ${ }^{1}$, IRINA P. ZAITSEVA ${ }^{1}$, ANDREY A. SKALNY ${ }^{3}$, \\ DEMETRIOS A. SPANDIDOS ${ }^{4}$, ARISTIDIS TSATSAKIS ${ }^{2,5}$, YULIA N. LOBANOVA ${ }^{3}$, \\ MARGARITA G. SKALNAYA ${ }^{1}$, MICHAEL ASCHNER ${ }^{2,6}$ and ALEXEY A. TINKOV ${ }^{1,2}$ \\ ${ }^{1}$ Laboratory of Biotechnology and Applied Bioelementology, Yaroslavl State University, Yaroslavl 150000; \\ ${ }^{2}$ Laboratory of Molecular Dietetics, IM Sechenov First Moscow State Medical University, Sechenov University, \\ Moscow 119435; ${ }^{3}$ Department of Medical Elementology, Peoples' Friendship University of Russia (RUDN University), \\ Moscow 117198, Russia; ${ }^{4}$ Laboratory of Clinical Virology; ${ }^{5}$ Center of Toxicology Science and Research, Medical School, \\ University of Crete, 70013 Heraklion, Greece; ${ }^{6}$ Department of Molecular Pharmacology,
}

Albert Einstein College of Medicine, New York, NY 10461, USA

Received December 29, 2020; Accepted February 15, 2021

DOI: $10.3892 /$ br.2021.1423

\begin{abstract}
The objective of the present study was to evaluate the circulating serum amino acid levels in children with attention deficit/hyperactivity disorder (ADHD). A total of 71 children with untreated ADHD and 31 neurotypical controls aged 7-14 years old were examined. Serum amino acid levels were evaluated using high-performance liquid chromatography (HPLC) with UV-detection. Laboratory quality control was performed with reference materials of human plasma amino acid levels. The obtained data demonstrated that children with ADHD were characterized by 29, 10 and 20\% lower serum histidine (His), glutamine (Gln) and proline (Pro) levels compared with neurotypical children, respectively. In contrast, circulating aspartate (Asp), glutamate (Glu) and hydroxyproline (Hypro) levels exceeded the respective control values by 7,7 and $42 \%$. Correspondingly, the Gln-to-Glu and Pro-to-Hypro ratios were $28 \%$ and $49 \%$, respectively, lower in ADHD cases compared with the controls. Total Gln/Glu levels were also significantly lower in ADHD patients. No significant group differences were observed between the groups in the other amino acids analyzed, including phenylalanine. Multiple linear regression analysis revealed significant associations between circulating serum Gln, lysine (Lys) (both negative) and Glu (positive) levels with total ADHD Rating Scale-IV scores. The observed alterations in Pro/Hypro and Gln/Glu levels and ratios are likely associated with the coexisting
\end{abstract}

Correspondence to: Dr Alexey A. Tinkov, Laboratory of Biotechnology and Applied Bioelementology, Yaroslavl State University, 14 Sovetskaya Street, Yaroslavl 150000, Russia E-mail: author@mail.com

Key words: attention deficit/hyperactivity disorder, hydroxyproline, glutamine, lysine, neurodevelopmental disorder connective tissue pathology and alterations in glutamatergic neurotransmission in ADHD, respectively. Altered circulating levels of His, Lys and Asp may also be implicated in ADHD pathogenesis. However, further in vivo and in vitro studies are required in order to investigate the detailed mechanisms linking amino acid metabolism with ADHD pathogenesis.

\section{Introduction}

Attention deficit/hyperactivity disorder (ADHD) is a neurodevelopmental disorder characterized by inattention and/or hyperactive-impulsivity that interferes with brain functioning or development. While contradictory, the existing data demonstrate that the prevalence of ADHD may be as high as 5.3\% and $2.5 \%$ in children/adolescents and adults, respectively (1). The effects of ADHD have a significant impact on the social life of patients throughout the entirety of their lives, starting from disruptive behavior, resulting in poor performance in standardized tests and impacted social interactions, which may lead to criminal behavior, substance abuse, lack of motivation, school exclusion and subsequent effects on professional development in adulthood (2). In addition, ADHD has also been found to be associated with lower a health-related quality of life (3). Taken together, these impairments result in high ADHD-associated economic costs (4).

ADHD is characterized by complex alterations in the neurobiology and neurochemistry, with impaired dopamine and norepinephrine signaling being the most prominent (5). Previously, it was also demonstrated that altered glutamatergic neurotransmission is involved in ADHD pathogenesis (6). Therefore, unraveling the potential underlying mechanisms implicated in ADHD pathogenesis is essential for improving our understanding of the disorder and further development of management strategies (5).

Amino acids serve a significant role in brain development and functioning (7). In particular, certain amino acids or their precursors, including glutamine, glutamate and $\gamma$ aminobutyric 
acid, are well-established to be involved in neuronal signaling as neurotransmitters (8). Correspondingly, disruption of amino acid metabolism results in significant neurological disorders, particularly in early ontogenesis (9).

Given the role of an altered neurochemistry in ADHD pathogenesis as well as the role of amino acids in neurodevelopment, it is posited that dysregulated amino acid metabolism may significantly interfere with ADHD. However, the existing data is rather contradictory. An earlier study by Bornstein et al (10) found significantly lower plasma levels of phenylalanine (Phe), tyrosine, tryptophan (Trp), His and isoleucine in patients with ADD compared with the healthy controls. Zavala et al (11) showed there was a significant decrease in plasma Phe and glutamine (Gln) levels, whereas plasma glycine (Gly) levels were found to be elevated in patients with ADHD. Improvement in ADHD symptoms was positively associated with tyrosine, Phe and Trp levels (12). At the same time, no significant alterations in blood and urinary levels of Trp, tyrosine and Phe levels were observed in children with ADHD (13). In view of these inconsistencies, as well as the use of amino acid supplementation in ADHD management, precise analyses of amino acid profiles in ADHD is required and may assist in reconciling these contrasting results. Thus, the objective of the present study was to evaluate the levels of circulating serum amino acid levels in children with ADHD.

\section{Materials and methods}

The present study was performed in accordance with the ethical principles of the Declaration of Helsinki and its amendments (14). The protocol of the present study was considered and approved by the Local Ethics Committee of Yaroslavl State University (Yaroslavl, Russia). Parents or legal representatives signed an informed consent forms for participation of their children in the present study prior to investigation. Examination and blood sample collection was performed only in the presence of the parents/guardians.

A total of 71 children (54 boys, 17 girls) diagnosed with ADHD aged 7-14 years old (8.4 2 2.6 years old) were enrolled in the present study. The diagnosis of ADHD (ICD-10: F90.0) and other neuropsychiatric disorders (exclusion criteria) were based on the clinical records of the outpatient department. ADHD was defined according to ICD-10 criteria, including inattention, hyperactivity and impulsivity ( $\geq 3$ symptoms of each) (15). Only patients that did not take any specific treatments for ADHD were included in the study, in order to avoid the confounding effects of any potential side effects of pharmacological treatment on amino acid metabolism.

In addition, 31 age $(8.0 \pm 2.9$ years old; age range $7-14$ years old) and sex-matched neurotypical children ( 24 boys, 7 girls) were also examined, and used as the control group. The absence of neuropsychiatric disorders was confirmed during annual medical examinations. No significant group differences in age $(\mathrm{P}=0.183)$ or sex $(\mathrm{P}=0.885)$ were observed between the groups.

Children and their parents were invited to participate in the study during the annual medical examinations. A total of $35 \%$ of all contacted subjects refused to take part in the study (36 out of 102 children and parents).

The parents of all the examined children completed the ADHD Rating Scale-IV for additional verification of the
ADHD diagnosis (16). Total ADHD Rating Scale-IV scores in the ADHD patients exceeded those in neurotypical children by a factor of $>2(14.9 \pm 9.4$ vs. $7.0 \pm 5.4, \mathrm{P}<0.001)$.

Whole blood samples were collected in the morning and after overnight fasting by cubital vein venipuncture using 9-ml Vacuette ${ }^{\circledR}$ tubes (Greiner Bio-One International AG). The samples were subsequently centrifuged for $10 \mathrm{~min}$ at $1,600 \mathrm{xg}$ at room temperature to obtain blood serum that was stored in Eppendorf tubes at $-18^{\circ} \mathrm{C}$ until required for analysis.

Evaluation of serum levels of alanine, arginine (Arg), asparagine, Asp, citrulline, glutamine (Gln), glutamate (Glu), Gly, histidine (His), hydroxyproline (Hypro), isoleucine, leucine, lysine (Lys), methionine, ornithine, Phe, proline (Pro), serine, taurine, threonine, Trp, and Val was performed by high-performance liquid chromatography (HPLC) with UV-detection at PerkinElmer S200 (PerkinElmer, Inc.) using a reverse phase Pico Tag Column for Free Amino Acid Analysis (3.9x300 mm) C18 (EMD Millipore).

Precolumn derivatization with phenylisothiocyanate reagent containing 7:1:1:1 (v/v) methanol:triethylamine:wa ter:phenylisothicyanate was performed prior to the analysis. Analysis was performed with aqueous sodium acetate and acetonitrile gradient mode with UV-detection at $240 \mathrm{~nm}$. The commercially available ClinCal ${ }^{\circledR}$ Plasma Calibrator, lyophil., for Amino Acids (lot. no. 10213; ClinCheck) calibrators were used for HPLC-system calibration.

Laboratory quality control was routinely performed with reference materials of human plasma amino acid levels using ClinChek ${ }^{\circledR}$ Plasma Control, lyophil., for Amino Acids, Levels I (cat. no. 10280) and II (cat. no. 10281). The obtained values for all amino acids fitted the certified control range specified by the manufacturer (ClinCheck). The recovery rates for the studied amino acids varied from 94-109\%.

Serum amino acid concentrations are expressed as $\mu \mathrm{mol} / 1$. In addition, total glutamatergic metabolite concentration (Glx), defined as a sum of Glu and Gln levels, was calculated as described previously (17). The obtained values of serum Gln, Glu, Hypro and Pro were used for calculating the Gln/Glu and Pro/Hypro ratios.

Statistical analysis was performed using Statistica version 10.1 (Statsoft, Inc.). Evaluation of data distribution performed using a Shapiro-Wilk test revealed skewed distribution of data on amino acid levels in the study groups. Therefore, the median and the respective interquartile range (IQR) boundaries were used as descriptive statistics. Raw data were log-transformed for subsequent processing. Group comparisons were performed using analysis of covariance (ANCOVA) with adjustment for age and sex as covariates and subsequent Bonferroni adjustments. Multiple linear regression was performed in order to evaluate the relative association between serum amino acid levels (independent predictors) and ADHD (dependent variable) after adjustment for age and sex. Correlation analysis was performed using a Spearman's rank correlation coefficient. $\mathrm{P}<0.05$ was considered to indicate a statistically significant difference, whereas $0.05<\mathrm{P}<0.1$ was considered nearly significant.

\section{Results}

The obtained data demonstrate that ADHD is associated with altered amino acid profiles in children. Evaluation of serum 
Table I. Essential amino acid levels in the serum of ADHD cases and neurotypical controls.

\begin{tabular}{lcccr}
\hline Amino acid & Control, $\mu \mathrm{M}^{\mathrm{a}}$ & ADHD, $\mu \mathrm{M}^{\mathrm{b}}$ & F-value & P-value \\
\hline Histidine & $85.0(50-120.6)$ & $60.7(45.2-94.6)$ & 3.140 & 0.081 \\
Isoleucine & $56.1(47.6-63.9)$ & $54.1(43.5-70.8)$ & 0.029 & 0.923 \\
Leucine & $118.6(103.6-133.7)$ & $117.5(96.2-138.5)$ & 0.105 & 0.862 \\
Lysine & $170.6(147.5-198.4)$ & $161.5(120.8-212.1)$ & 1.242 & 0.360 \\
Methionine & $54.2(46.2-69.1)$ & $51.6(44.1-60.2)$ & 0.416 & 0.396 \\
Phenylalanine & $55.8(47.5-68.2)$ & $57.8(48.4-66.4)$ & 0.009 & 0.923 \\
Threonine & $108.9(90.5-129)$ & $111.4(86.8-143.1)$ & 0.768 & 0.605 \\
Tryptophan & $56.5(43.3-70.6)$ & $58.1(44.6-71.5)$ & 0.011 & 0.794 \\
Valine & $167.2(136.9-212.3)$ & $167.2(128-223.4)$ & 0.060 & 0.907 \\
\hline
\end{tabular}

${ }^{a}$ Data are presented as the median (inter-quartile range). ${ }^{\mathrm{b}} \mathrm{ADHD}$, attention deficit/hyperactivity disorder.
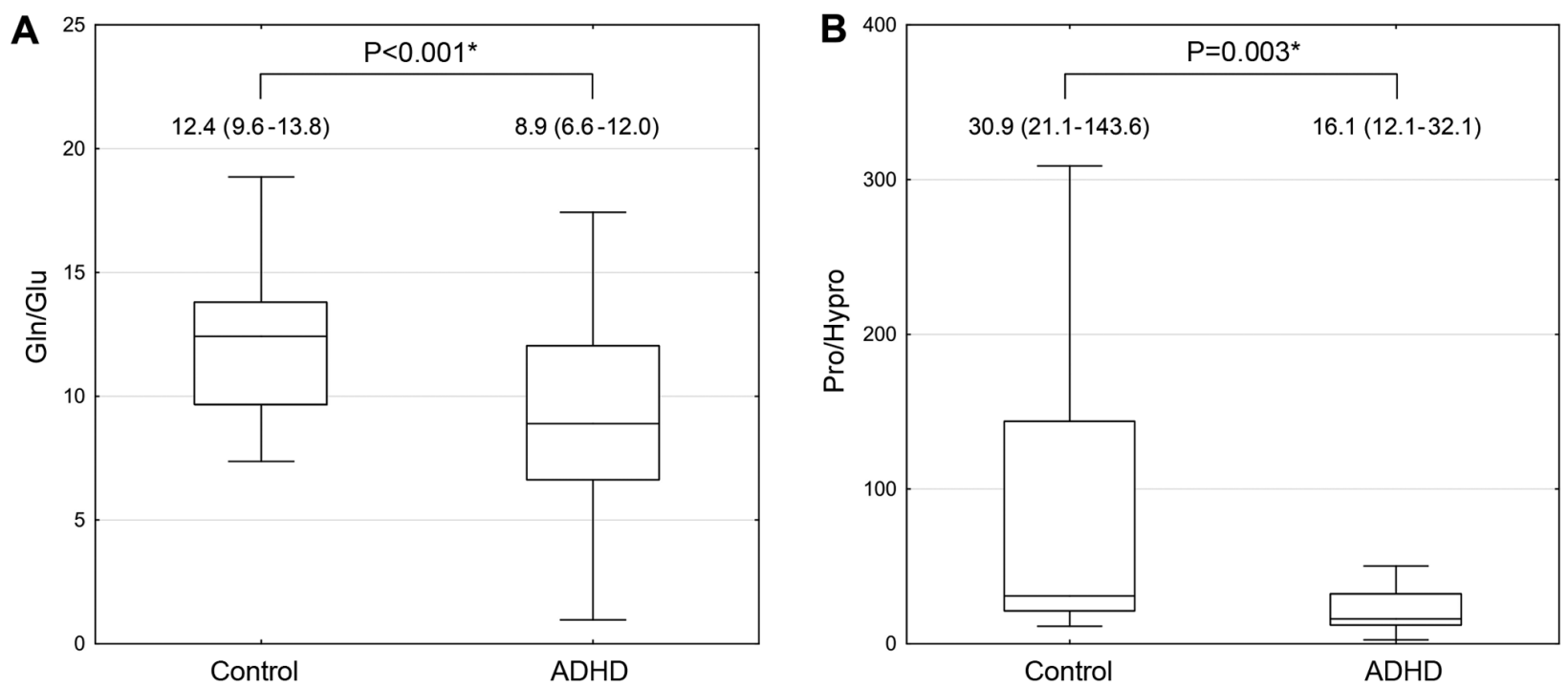

Figure 1. Serum Gln/Glu and Pro/Hypro ratios in children with ADHD compared with the healthy controls. (A) Serum Gln/Glu ratio. (B) Serum Pro/Hypro ratio. Data are expressed as the median (line), inter-quartile ranges (box) and non-outlier ranges (whiskers). *P<0.05. Gln, glutamine; Glu, glutamine; Pro, proline; Hypro; hydroxyproline.

essential amino acids revealed $29 \%$ lower His levels in ADHD patients compared with the neurotypical controls (Table I). No significant group differences in other essential amino acids levels were observed.

Greater differences were observed in the levels of conditionally essential and non-essential amino acids (Table II). Particularly, serum Asp and Glu levels were 7\% higher compared with the healthy controls, although the difference was significant only in the case of Glu levels. In contrast, serum GIn and Pro concentrations were $10 \%$ and $20 \%$ lower in ADHD cases compared with the neurotypical controls, respectively. In corroboration with the overall decrease in serum Gln levels, total Glx concentration (Glu+Gln) was also $12 \%$ lower in children with ADHD [425.5 (351.5-533.6) $\mu \mathrm{M}]$ compared with the control values [483.1 (406.0-577.4) $\mu \mathrm{M}](\mathrm{F}=4.007 ; \mathrm{P}=0.048)$.

Amongst the amino acid derivatives analyzed, only serum Hypro concentrations were significantly elevated, being $42 \%$ higher in ADHD cases compared with the healthy controls.
Given the observed group differences between serum Gln and Glu levels, as well as the levels of Pro and its derivative Hypro, Gln-to-Glu and Pro-to-Hypro ratios were evaluated (Fig. 1). The obtained data demonstrate that Gln/Glu values in ADHD cases were $28 \%$ lower compared with the healthy children $(\mathrm{F}=14.202)$. In turn, ADHD patients had almost 2-fold lower levels of Pro/Hypro levels compared with the neurotypical controls $(\mathrm{F}=8.936)$.

Correlation analysis demonstrated that serum Hypro and Glu concentrations were correlated significantly $(\mathrm{r}=0.270$; $\mathrm{P}=0.006)$ and nearly significantly $(\mathrm{r}=0.194 ; \mathrm{P}=0.051)$ with total ADHD-RS scores, respectively. Concomitantly, circulating Gln levels ( $\mathrm{r}=-0.207 ; \mathrm{P}=0.037)$ as well as $\mathrm{Gln} / \mathrm{Glu}$ ratio $(\mathrm{r}=-0.376 ; \mathrm{P}<0.001)$ were characterized by a significant direct correlation with the latter.

Multiple regression analysis was also performed in order to determine if there was an independent association between the serum amino acid levels and total ADHD-RS scores (Table III). In a crude model incorporating all amino acids analyzed, serum 
Table II. Conditionally essential and non-essential amino acid levels the serum of children with ADHD and healthy controls.

\begin{tabular}{lcccc}
\hline Amino acid & Control, $\mu \mathrm{M}^{\mathrm{b}}$ & ADHD, $\mu \mathrm{M}^{\mathrm{b}}$ & F-value & P-value \\
\hline Alanine & $323.5(248.3-395.5)$ & $342.7(298.1-401.9)$ & 0.779 & 0.342 \\
Arginine & $68.9(55.7-85.8)$ & $66.5(55.9-81.6)$ & 0.008 & 0.988 \\
Asparagine & $77.8(67.7-85.0)$ & $78.5(64.8-91.6)$ & 0.032 & 0.872 \\
Aspartate & $13.9(10.5-17.9)$ & $14.9(11.1-20.3)$ & 2.335 & 0.103 \\
Glutamine & $433.4(379.5-534.4)$ & $389(312.8-474.7)$ & 5.935 & $0.024^{\mathrm{a}}$ \\
Glutamate & $39.3(27.2-46.5)$ & $42.0(34.2-53.5)$ & 4.130 & $0.039^{\mathrm{a}}$ \\
Glycine & $418.4(374.7-455.2)$ & $396.7(340.5-469.7)$ & 0.181 & 0.724 \\
Proline & $316.3(219.0-418.1)$ & $254.6(207.2-319.8)$ & 3.690 & $0.045^{\mathrm{a}}$ \\
Serine & $83.2(68.7-92.8)$ & $82.5(66.1-97.0)$ & 0.034 & 0.934 \\
Taurine & $64.5(50.6-82.1)$ & $66.4(55.6-82.7)$ & 0.709 & 0.416 \\
Tyrosine & $82.9(72.6-98.3)$ & $85.5(75.7-101.0)$ & 0.090 & 0.718 \\
Citrulline & $54.1(42.1-70.1)$ & $50.9(42.4-64.9)$ & 0.548 & 0.541 \\
Ornithine & $58.2(46.5-71.1)$ & $60.4(45.8-83.0)$ & 0.068 & 0.882 \\
Phosphoserine & $54.7(26.9-89.8)$ & $50.3(31.5-66.1)$ & 0.502 & 0.435 \\
Hydroxyproline & $11.4(1.6-18.7)$ & $16.2(11.3-20.8)$ & 4.752 & $0.018^{\mathrm{a}}$ \\
\hline
\end{tabular}

${ }^{\mathrm{a}} \mathrm{P}<0.05 ;{ }^{\mathrm{b}} \mathrm{Data}$ are presented as the median (inter-quartile range). ADHD, attention deficit/hyperactivity disorder.

Table III. Multivariate linear regression analysis of the association between serum amino acid levels (independent predictor) and attention deficit/hyperactivity disorder Rating Scale-IV scores (dependent variable).

\begin{tabular}{lrrrrr}
\hline & \multicolumn{2}{c}{ Model 1 } & & \multicolumn{2}{c}{ Model 2 } \\
\cline { 2 - 3 } \cline { 5 - 6 } Amino acid & $\beta$ & P-value & & $\beta$ & P-value \\
\hline Ala & 0.140 & 0.460 & & 0.050 & 0.724 \\
Asp & -0.009 & 0.965 & & -0.077 & 0.596 \\
Gln & -0.353 & $0.022^{\mathrm{a}}$ & -0.370 & $0.002^{\mathrm{b}}$ \\
Glu & 0.258 & 0.206 & & 0.350 & $0.029^{\mathrm{a}}$ \\
His & -0.150 & 0.218 & & -0.057 & 0.583 \\
Hypro & 0.227 & 0.111 & 0.211 & 0.065 \\
Lys & -0.527 & $0.027^{\mathrm{a}}$ & -0.339 & $0.021^{\mathrm{a}}$ \\
Orn & 0.281 & 0.087 & 0.161 & 0.248 \\
Pro & -0.051 & 0.643 & -0.082 & 0.391 \\
Thr & 0.327 & 0.081 & 0.169 & 0.214 \\
Multiple R & 0.576 & 0.518 & & \\
Multiple R & & 0.332 & 0.268 & & \\
Adjusted $\mathrm{R}^{2}$ & 0.100 & 0.170 & & \\
P for the model & 0.116 & $0.004^{\mathrm{b}}$ & & \\
\hline
\end{tabular}

${ }^{\mathrm{a}} \mathrm{P}<0.05,{ }^{\mathrm{b}} \mathrm{P}<0.01$. Data are expressed as the regression coefficient $(\beta)$ and the respective $\mathrm{P}$-value.

Gln and Lys levels were found to be inversely associated with total ADHD-RS scores. The overall model trended towards statistically significant $(\mathrm{P}=0.072)$, accounting for only $12 \%$ of total ADHD-RS scores. In a model incorporating amino acids considered to be significantly and almost significantly associated with ADHD scores (Model 2), serum Gln and Lys remained significantly associated with ADHD, whereas circulating Glu levels appeared to be positively associated with total ADHD-RS scores. Serum Hypro levels were almost significantly associated with ADHD scores. Although the predictive value of Model 2 was significant, it accounted for only $17 \%$ of score variability. Neither age nor sex were associated significantly with ADHD in both regression models.

\section{Discussion}

The results of the present study demonstrated that children with ADHD were characterized by distinct amino acid profiles compared with the controls, indicative of predominant alteration in Glu, Pro and Lys metabolism. Significant group differences in Glu/Gln ratio may be indicative of altered neurotransmission in children with ADHD, whereas high Hypro levels and a high Hypro/Pro ratio may be considered as a marker of collagen catabolism and connective tissue pathology.

Existing data demonstrate that alterations in glutamatergic signaling may contribute significantly to ADHD pathogenesis (6). Specifically, it has been demonstrated that ADHD patients are characterized by significantly lower Gln plus Glu levels in basal ganglia (18), including in the striatum (19). An increase in anterior cingulate cortex Glu content was also associated with hyperactivity and impulsivity in adult ADHD patients (20). Pertinent genome-wide analyses for ADHD risk genes revealed altered expression profiles of genes associated with glutamatergic neurotransmission (21). Correspondingly, altered AMPAR-mediated transmission in pyramidal neurons of the prefrontal cortex was also found to be associated with ADHD in an experimental rat model (22).

In view of the role of Hypro as a marker of connective tissue pathology (23), the earlier proposed association between 
ADHD and joint hypermobility (24) may underlie the observed increase in plasma Hypro levels in children with ADHD. Particularly, joint hypermobility was found to be $>2$-fold more prevalent in ADHD subjects compared with the reference population (25). Moreover, connective tissue disorders are also closely associated with other neurodevelopmental disorders (26). These data also corroborate our earlier findings on increased Hypro levels in autism spectrum disorder (27) and cerebral palsy (28). In addition, in ADHD subjects, Pro levels were found to be reduced with a decreased Pro/Hypro ratio, and levels were found to be inversely associated with S100B and positively related to IL-10 levels (29), indicative of the potential contribution of dysregulated Pro metabolism in this neurodevelopmental disorder.

The results of serum amino acid profiling also demonstrated group differences in serum His, Asp and Lys levels between ADHD patients and neurotypical controls. Although an essential role of His in brain physiology has been demonstrated (30), data on the relationship between ADHD and His metabolism are insufficient. His supplementation was shown to reduce fatigue and improve mental performance in subjects with high fatigue and sleep disruption scores (31). In turn, experimental data demonstrated that a His-deficient diet resulted in formation of anxiety-like behaviors in mice through reduction of brain histamine levels (32). In turn, increased serum Asp levels in patients with ADHD generally correspond with an earlier observed higher Asp intake in ADHD patients (33).

It is noteworthy that the present study revealed a significant association between lower serum Lys levels and ADHD. Despite the role of Lys metabolism in brain physiology (34), earlier data on the involvement of Lys in ADHD pathophysiology are lacking. Nonetheless, Lys supplementation has been used in ADHD management (35), based on observations that Lys as well as Arg treatments reduce anxiety in stressed adults characterized by high cortisol levels (36).

In contrast to earlier reports $(11,12)$, significant group differences in tyrosine, phenylalanine or Trp levels were not observed in the present study, in agreement with a study by Bergwerff et al (13) who did not reveal any significant group differences in serum and urinary levels of the studied amino acids (13).

The present study also has several limitations. First, this was a cross-sectional study involving a relatively small number of patients, and studying larger cohorts of both cases and controls may improve the statistical power of the results. Second, follow-up design of the study with evaluation of ADHD incidence and severity would be beneficial to provide an insight into the causal relationships between ADHD and the observed alterations in amino acid metabolism. Third, although the results demonstrate the potential alterations of amino acid-derived neurotransmitters in patients with ADHD, serum levels do not necessarily reflect brain levels of amino acids and their derivatives. Therefore, evaluation of cerebrospinal fluid amino acid levels or the use of techniques for direct brain metabolite assessment, such as proton magnetic resonance spectroscopy, would assist in highlighting ADHD-associated alterations in metabolism of brain amino acids and neurotransmitters.

In conclusion, the results of the present study demonstrated significant alterations in the serum amino acid profile of children with ADHD. The observed alterations of Pro/Hypro and Gln/Glu levels and their ratios may be associated with the coexisting connective tissue pathology and alterations of glutamatergic neurotransmission in ADHD, respectively. Altered circulating levels of His, Lys and Asp may also be implicated in the pathogenesis of ADHD. However, further in vitro and in vivo studies are required to investigate the specific underlying mechanisms linking amino acid metabolism with ADHD pathogenesis.

\section{Acknowledgements}

Not applicable.

\section{Funding}

The present study was supported by funding from Russian Foundation for Basic Research (RFBR) (grant no. 19-013-00528).

\section{Availability of data and materials}

The datasets used and/or analyzed during the present study are available from the corresponding author on reasonable request.

\section{Authors' contributions}

AVS, MGS and AAT conceived the study. AVS, AAT, AT, DAS and MA designed the study. AVS, AAT, AAS, ALM, IPZ, YNL and MGS performed the experiments. ALM, IPZ, AVS, AT and DAS analyzed the data. ALM, AAT, AAS, IPZ, YNL, AVS, MGS, AT, DAS and MA wrote and edited the manuscript. All authors have read and approved the final manuscript. AVS, MGS and AAT confirm the authenticity of all the raw data.

\section{Ethics approval and consent to participate}

The present study was performed in accordance with the ethical principles of the Declaration of Helsinki and its amendments (2013). The protocol of the present study was considered and approved by the Local Ethics Committee (Yaroslavl State University, Yaroslavl, Russia). Parents or legal representatives signed an informed consent form for participation of their children in the present study prior to investigation. Examination and blood sample collection was performed only in the presence of the parents/guardians.

\section{Patient consent for publication}

Not applicable.

\section{Competing interests}

DAS is the Editor-in-Chief for the journal, but had no personal involvement in the reviewing process, or any influence in terms of adjudicating on the final decision, for this article. The other authors declare that they have no competing interests.

\section{References}

1. Posner J, Polanczyk GV and Sonuga-Barke E: Attention-deficit hyperactivity disorder. Lancet 395: 450-462, 2020 
2. Harpin VA: The effect of ADHD on the life of an individual, their family, and community from preschool to adult life. Arch Dis Child 90 (Suppl 1): 2-7, 2005.

3. Peasgood T, Bhardwaj A, Biggs K, Brazier JE, Coghill D, Cooper CL, Daley D, De Silva C, Harpin V, Hodgkins P, et al: The impact of ADHD on the health and well-being of ADHD children and their siblings. Eur Child Adolesc Psychiatry 25: 1217-1231, 2016.

4. Barkley RA: The high economic costs associated with ADHD. ADHD Rep 28: 10-12, 2020.

5. Mehta TR, Monegro A, Nene Y, Fayyaz M and Bollu PC: Neurobiology of ADHD: A review. Curr Dev Disord Rep 6: 235-240, 2019

6. Levy F and de Leon J: Dopamine ADHD/OCD theories: Is glutamine part of the story? Neurotransmitter (Houst) 2: e891, 2015

7. Wu G: Functional amino acids in growth, reproduction, and health. Adv Nutr 1: 31-37, 2010.

8. ValenzuelaCF,PugliaMPandZuccaS: Focus on: Neurotransmitter systems. Alcohol Res Health 34: 106-120, 2011

9. Saudubray JM and Garcia-Cazorla A: An overview of inborn errors of metabolism affecting the brain: From neurodevelopment to neurodegenerative disorders. Dialogues Clin Neurosci 20 301-325, 2018

10. Bornstein RA, Baker GB, Carroll A, King G, Wong JT and Douglass AB: Plasma amino acids in attention deficit disorder Psychiatry Res 33: 301-306, 1990.

11. Zavala M, Castejón HV, Ortega PA, Castejón OJ, Marcano de Hidalgo A and Montiel N: Imbalance of plasma amino acids in patients with autism and subjects with attention deficit/hyperactivity disorder. Rev Neurol 33: 401-408, 2001.

12. Stern M, Perez L, Johnstone J, Gracious B, Leung B, Tost G, Arnold E, Hatsu I and Kopec R: Using Metabolomics to classify the underlying effects of multi-nutrient supplementation in ADHD youth. Curr Dev Nutr 4 (Suppl 2): 1351, 2020.

13. Bergwerff CE, Luman M, Blom HJ and Oosterlaan J: No tryptophan, tyrosine and phenylalanine abnormalities in children with attention-deficit/hyperactivity disorder. PLoS One 11: e0151100, 2016.

14. General Assembly of the World Medical Association: World Medical Association Declaration of Helsinki: Ethical principles for medical research involving human subjects. J Am Coll Dent 81: 14-18, 2014.

15. International Statistical Classification of Diseases and Related Health Problems 10th Revision (ICD-10): Chapter V Mental and behavioural disorders (F00-F99). Behavioural and emotional disorders with onset usually occurring in childhood and adolescence (F90-F98). https://icd.who.int/browse10/2019/en\#/ F90-F98. Accessed December 8, 2020.

16. DuPaul GJ, Power TJ, Anastopoulos AD and Reid R: ADHD Rating Scale-IV: Checklists, norms, and clinical interpretation. Guilford Press, 1998.

17. Goff DC, Hennen J, Lyoo IK, Tsai G, Wald LL, Evins AE, Yurgelun-Todd DA and Renshaw PF: Modulation of brain and serum glutamatergic concentrations following a switch from conventional neuroleptics to olanzapine. Biol Psychiatry 51: 493-497, 2002.

18. Maltezos S, Horder J, Coghlan S, Skirrow C, O'Gorman R, Lavender TJ, Mendez MA, Mehta M, Daly E, Xenitidis K, et al: Glutamate/glutamine and neuronal integrity in adults with ADHD: A proton MRS study. Transl Psychiatry 4: e373, 2014.

19. Carrey NJ, MacMaster FP, Gaudet L and Schmidt MH: Striatal creatine and glutamate/glutamine in attention-deficit/hyperactivity disorder. J Child Adolesc Psychopharmacol 17: 11-17, 2007.

20. Bauer J, Werner A, Kohl W, Kugel H, Shushakova A, Pedersen A and Ohrmann P: Hyperactivity and impulsivity in adult attention-deficit/hyperactivity disorder is related to glutamatergic dysfunction in the anterior cingulate cortex. World J Biol Psychiatry 19: 538-546, 2018.
21. Lesch KP, Merker S, Reif A and Novak M: Dances with black widow spiders: Dysregulation of glutamate signalling enters centre stage in ADHD. Eur Neuropsychopharmacol 23: 479-491, 2013.

22. Cheng J,Liu A, Shi MY and Yan Z: Disrupted glutamatergic transmission in prefrontal cortex contributes to behavioral abnormality in an animal model of ADHD. Neuropsychopharmacology 42 2096-2104, 2017

23. Srivastava AK, Khare P, Nagar HK, Raghuwanshi N and Srivastava R: Hydroxyproline: A potential biochemical marker and its role in the pathogenesis of different diseases. Curr Protein Pept Sci 17: 596-602, 2016.

24. Baeza-VelascoC,SinibaldiLandCastoriM:Attention-deficit/hyperactivity disorder, joint hypermobility-related disorders and pain: Expanding body-mind connections to the developmental age. Atten Defic Hyperact Disord 10: 163-175, 2018.

25. Doğan ŞK, Taner Y and Evcik D: Benign joint hypermobility syndrome in patients with attention deficit/hyperactivity disorders. Arch Rheumatol 26: 187-192, 2011.

26. Baeza-Velasco C, Grahame R and Bravo JF: A connective tissue disorder may underlie ESSENCE problems in childhood. Res Dev Disabil 60: 232-242, 2017.

27. Skalny AV, Skalny AA, Lobanova YN, Korobeinikova TV, Ajsuvakova OP, Notova SV, Burtseva TI, Skalnaya MG and Tinkov AA: Serum amino acid spectrum in children with autism spectrum disorder (ASD). Res Autism Spectr Disord 77: 101605, 2020.

28. Tinkov AA, Skalnaya MG and Skalny AV: Serum trace element and amino acid profile in children with cerebral palsy. J Trace Elem Med Biol 64: 126685, 2021.

29. Oades RD, Dauvermann MR, Schimmelmann BG, Schwarz MJ and Myint AM: Attention-deficit hyperactivity disorder (ADHD) and glial integrity: S100B, cytokines and kynurenine metabolism - effects of medication. Behav Brain Funct 6: 29, 2010.

30. Holeček M: Histidine in health and disease: Metabolism, physiological importance, and use as a supplement. Nutrients 12: 848, 2020.

31. Sasahara I, Fujimura N, Nozawa Y, Furuhata Y and Sato H: The effect of histidine on mental fatigue and cognitive performance in subjects with high fatigue and sleep disruption scores. Physiol Behav 147: 238-244, 2015

32. Yoshikawa T, Nakamura T, Shibakusa T, Sugita M, Naganuma F, Iida T, Miura Y, Mohsen A, Harada R and Yanai K: Insufficient intake of L-histidine reduces brain histamine and causes anxiety-like behaviors in male mice. J Nutr 144: 1637-1641, 2014.

33. Holton KF, Johnstone JM, Brandley ET and Nigg JT: Evaluation of dietary intake in children and college students with and without attention-deficit/hyperactivity disorder. Nutr Neurosci 22: 664-677, 2019

34. Hallen A, Jamie JF and Cooper AJ: Lysine metabolism in mammalian brain: An update on the importance of recent discoveries. Amino Acids 45: 1249-1272, 2013.

35. Mikirova NA, Rogers AM, Taylor PR, Hunninghake RE, Miranda-Massari JR and Gonzalez MJ: Metabolic correction for attention deficit/hyperactivity disorder: A biochemical-physiological therapeutic approach. Funct Food Health Dis 3: 1-20, 2013.

36. Smriga M, Ando T, Akutsu M, Furukawa Y, Miwa $\mathrm{K}$ and Morinaga Y: Oral treatment with L-lysine and L-arginine reduces anxiety and basal cortisol levels in healthy humans. Biomed Res 28: 85-90, 2007.

This work is licensed under a Creative Commons Attribution-NonCommercial-NoDerivatives 4.0 International (CC BY-NC-ND 4.0) License. 